\title{
Video consultations as add-on to standard care among patients with type 2 diabetes not responding to standard regimens: a randomized controlled trial
}

\author{
Caroline Raun Hansen', Hans Perrild', Birgitte Gade Koefoed ${ }^{\mathbf{2}}$ and Mette Zander ${ }^{1}$ \\ ${ }^{1}$ Department of Endocrinology, Bispebjerg University Hospital, Copenhagen NV, Denmark and ${ }^{2}$ Healthcare Centre \\ Nørrebro, Copenhagen NV, Denmark
}

Correspondence should be addressed to $\mathrm{C}$ R Hansen Email

chan0294@regionh.dk

\begin{abstract}
Objective: To examine whether video consultations preceded by measurements of blood glucose, weight and blood pressure as add-on to standard care could contribute to achieving and maintaining good diabetes control among patients with poorly regulated type 2 diabetes (T2D).

Design: Randomized controlled trial.

Methods: 165 patients with T2D were randomized 1:1 to telemedicine intervention as add-on to clinic-based care or control (clinic-based care). The intervention consisted of monthly video conferences with a nurse via a tablet computer and lasted for 32 weeks. Regularly self-monitored measurements of blood sugar, blood pressure and weight were uploaded and visible to patient and nurse. Both groups were followed up six months after the end of the intervention period.

Primary endpoint: HbA1c after eight months.

Results: Video conferences preceded by uploads of measurements as add-on to clinic-based care led to a significant reduction of $\mathrm{HbA} 1 \mathrm{c}$ compared to that in standard care $(0.69 \%$ vs $0.18 \%, P=0.022)$. However, at six-month follow-up, the inter-group difference in HbA1c-reduction was no longer significant. Non-completers had higher HbA1c levels at baseline and a lower degree of education.

Conclusion: Video consultations preceded by uploading relevant measurements can lead to clinically and statistically significant improvements in glycemic control among patients who have not responded to standard regimens. However, continuing effort and attention are essential as the effect does not persist when intervention ends. Furthermore, future studies should focus on differentiation as the most vulnerable patients are at greater risk of non-adherence.
\end{abstract}

\section{Introduction}

The prevalence of diabetes is increasing worldwide (1) rendering grave consequences to both individuals and society. Patients with type 2 diabetes (T2D) who do not respond to standard treatment and disease management programs constitute a special challenge. Inequality in the health care system is well known. Higher SES populations are more prone to attend disease management courses
(C) 2017 European Society of Endocrinology Printed in Great Britain than low SES populations. In lower social groups a higher incidence of type 2 diabetes $(2,3,4,5)$ and increased risk of developing complications has been described (6). Vulnerability in relation to disease does not have a clear definition but refers to patients with difficulty in coping with disease. Low socioeconomic status does not per se make a patient vulnerable but as low education

Published by Bioscientifica Ltd. 
correlates to mortality and comorbidity, vulnerable patients are presumably more frequent among lower socioeconomic groups. Ethnical minority groups have higher prevalence of diabetes $(7,8)$ and poorer diabetes control (9). Immigrants often have lower socioeconomic status (10) and poorer self-perceived health relating to increased mortality and morbidity (11). Thus, vulnerable patients might also be overrepresented among ethnical minority groups. To improve care in vulnerable patients with diabetes, recent studies have focused on alternative programs for diabetes control, including peer-led diabetes education and telephone contacts $(12,13,14)$; however, research is still needed for improving diabetes control in this population. Telemedicine is defined as the use of medical information exchanged from one site to another via electronic communications to improve a patient's clinical health status (15). It ranges from telephone calls and text messages to apps on smartphones or internet-based solutions. In line with the technological development and with the easier access to technological solutions, the use of telemedicine is increasing rapidly.

The IDEATel study compared a wide spectrum of telehealth interventions ranging from video conferences to an educational website to standard care. The study was performed in a medically underserved area. In this study, participants with lower socio economic status (SES) benefited as much as higher SES participants (16). Thus, it was found that interventions may have the potential to reduce disparities in diabetes management (17).

In line with the IDEATel, other studies using tele technologies have addressed difficulties in distance or understaffing $(18,19,20,21)$. The level of care in the Capital Region of Denmark is high. All patients with T2D are offered participation in a diabetes management program (22). In addition to the standard programs, our local healthcare center offers group-based courses for ethnic minorities in Arabic, Urdu and Turkish.

In contrast to IDEALtel and other studies, our study was designed to examine the effect of video consultations as add-on to clinic-based care in a vulnerable population of patients with T2D who had already been offered a high level of standard care as the Capital Region of Denmark is not medically underserved. Thus, our study focused on patients apparently incapable of coping with their disease.

\section{Objectives}

To determine whether monthly video consultations with a nurse preceded by regular measurements of blood sugar, blood pressure and weight uploaded to a common database in addition to clinic-based care could improve the glycemic regulation of patients with poorly regulated T2D, who had previously attended a disease management program. Moreover, to determine if a given effect would last after intervention.

\section{Subjects and methods}

We have previously described the study in detail (23).

\section{Trial design}

The study was cross-sectional and done in cooperation between the City of Copenhagen and Bispebjerg University Hospital.

One hundred sixty-five patients were randomized 1:1 to usual care or telemedicine intervention as add-on to clinic-based care. Participants were randomized by drawing a sealed envelope containing a number. Depending on the number, the patients were allocated to either intervention or control. Due to the nature of the study, it was not possible to blind subjects.

\section{Participants}

We recruited participants from the outpatient department of three hospitals in Copenhagen, Denmark and from the local health care center. All patients had completed a 4- to 6-month diabetes management program prior to participation in the study, but had not reached their goal in spite of this. Participants were not offered economic compensation. Most participants came from Bispebjerg University Hospital, Copenhagen, Denmark. Bispebjerg is known for having a composite catchment area stretching from high-income districts, where the majority is well educated, to lower income districts, where education is sparse. Many are unemployed and suffer from chronic diseases, and a high proportion originates from nonWestern countries.

\section{Eligibility}

Danish speaking inhabitants of the City of Copenhagen with T2D and a history of participation in a diabetes management program at least six months prior to inclusion were eligible. Additional inclusion criteria were willingness and ability to participate, $\mathrm{HbA1c}>7.5 \%$ 
(59 mmol $/ \mathrm{mol})$, body mass index (BMI) $>25 \mathrm{~kg} / \mathrm{m}^{2}$ and age $30-75$ years.

Exclusion criteria were terminal disease with an expected lifespan of $<6$ months or need of an interpreter.

\section{Study settings}

An endocrinologist and a study nurse based at Bispebjerg University Hospital enrolled participants.

\section{Intervention}

The intervention consisted of monthly video conferences with a health care center nurse via a tablet computer. Participants regularly uploaded measurements of blood sugar, blood pressure and weight directly from the meters via Bluetooth or USB jack to a tablet computer (23). Measurements were visible to both participant and the health care center nurse and formed the basis of the consultations. A Windows 7 tablet with a $3 \mathrm{G}$ modem was handed out to all participants in the intervention group during the study.

The intervention was based upon the principles of empowerment and aimed toward participants being responsible of self-management and control of their disease. An interview guide carefully described the topics and the timeline of the consultations. It was made by the nurses in cooperation with the medical chief of the health care center. All nurses were experienced in rehabilitation of patients with type 2 diabetes and/ or cardiovascular disease. Furthermore, all nurses had completed an educational program in type 2 diabetes prior to commencing the study. During this program, the consequences of hypoglycemia were addressed as hypoglycemia should be avoided in patients with macrovascular complications (24). Nurses ensured that patients would contact the doctor responsible for diabetes control in case of hypoglycemic incidents.

A pilot study was performed prior to commencing the study. Here, logistical and technical difficulties were found and addressed. Moreover, the pilot study was a way of training the art of video consultations.

The intervention was an add-on to clinic-based care. Clinic-based care consisted of a doctor's visits every 3-6 months. Some participants were seen at an outpatient department and others by their general practitioner. The goal HbA1c was set by the doctor responsible for treatment (not part of the study team). The study had no influence on the usual place of control. The study team did not manage medication. However, special attention was drawn to patients with macrovascular complications to reduce the risk of hypoglycemia.

The intervention lasted eight months and participants were followed up for six months after intervention. The control group followed usual care throughout all 14 months. The intervention is fully described in the protocol paper (23).

\section{Outcomes}

The primary outcome was HbA1c at eight months (end of intervention). We also assessed changes between HbA1c from baseline to 8 months thereby taking into account the impact of baseline values. Moreover, we assessed the differences between 8 and 14 months, as we were interested in differences after the end of intervention.

Secondary outcomes were changes in blood pressure, lipid levels, BMI, waist/hip ratio, quality of life assessed by a modified version of the Short Form 36, version 1 (SF-36) questionnaire and diabetes-related hospital admissions. Moreover, we assessed changes in insulin doses and the fraction of patients treated with insulin. The randomization status was not blinded for the outcome assessor.

\section{Sample size}

The study was powered to detect an inter-group difference in $\mathrm{HbA} 1 \mathrm{c}$ of $0.5 \%(5.5 \mathrm{mmol} / \mathrm{mol})$. Using a standard deviation of 0.9 , test strength of 0.9 and a $5 \%$ level of significance, we found that 137 subjects were to be included. We increased the sample size to 165 anticipating a dropout rate of $20 \%$.

\section{Tests}

Baseline tests: Hemoglobin, creatinine, HbA1c, lipids, urine albumin/creatinine ratio, blood pressure, BMI, waist/hip ratio and the Short Form 36 questionnaire (SF-36).

Four months: HbA1c.

Eight months: Hemoglobin, creatinine, HbA1c, lipids, blood pressure, BMI, waist/hip ratio and SF-36 questionnaire.

Follow-up (14 months): HbA1c, blood pressure, BMI and waist/hip ratio. 


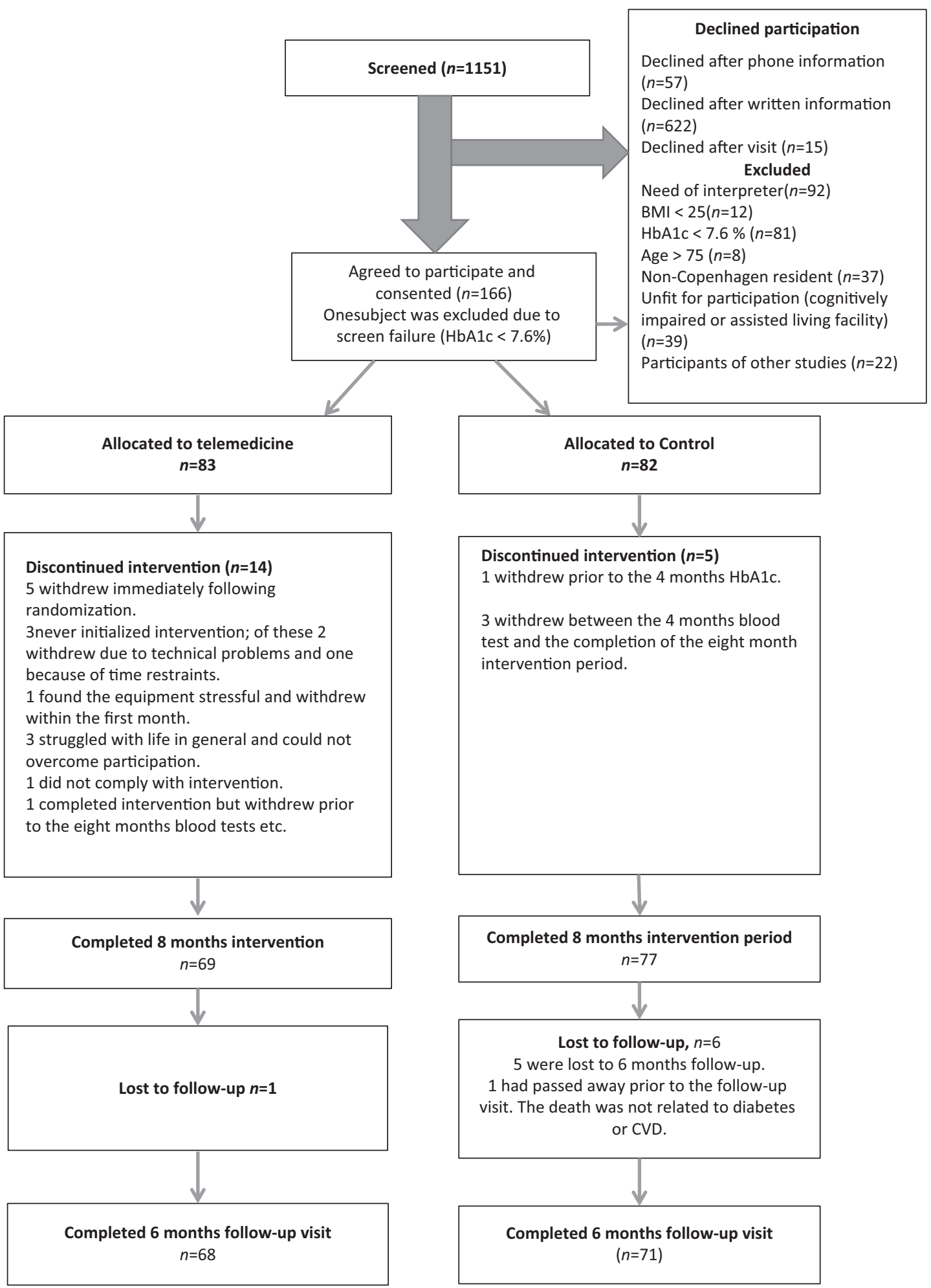

Figure 1

Participant flow. 


\section{Statistical methods}

Descriptive statistics, ANOVA, normality tests and paired $T$-tests were done using $\mathrm{R}$ Studio statistical software, version 3.1.1, mixed-models analyses in SAS statistical software, version 9.4 (SAS Institute, Cary, NC, USA). Baseline data of the two groups were compared. Chisquare (Yates correction when cell numbers <10) was used for categorical data and unpaired $T$-test was used for continuous data. Normality tests for continuous data were performed by a QQ test.

The primary outcome (HbA1c at 8 months) and secondary outcomes (lipids, blood pressure, BMI and waist/hip ratio) were assessed using mixed models. Furthermore, the difference in HbA1c between baseline and eight months was estimated using two-way ANOVA, adjusting for baseline HbA1c and the presence of complications. The analyses were made both with and without intention to treat, the latter being the primary analysis.

\section{Trial registration and ethics}

All participants signed a written consent form prior to inclusion in the study. The study was approved by the Research Ethics Committee, the Capital Region of Denmark (H-2-2011-158), and by the Danish Data Protection Agency (2011-41-6999). It was conducted in accordance to the Helsinki declaration. ClinicalTrials.gov registration number: NCT01688778.

\section{Results}

Recruitment occurred from April 2012 to February 2014, and the study was finalized by April 2015 . We screened

Table 1 Characteristics at baseline.

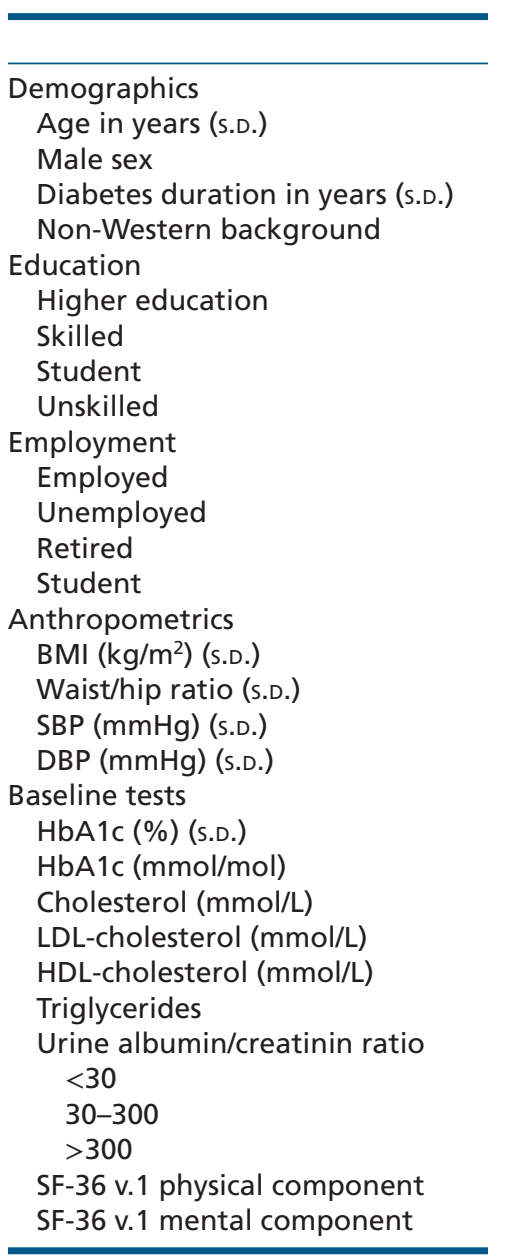

\begin{tabular}{c}
\hline Control $(n=82)$ \\
$58.3(9.3)$ \\
$53(65 \%)$ \\
$12.5(7.3)$ \\
$21(26 \%)$ \\
$15(18 \%)$ \\
$36(44 \%)$ \\
$2(2.5 \%)$ \\
$29(35.5 \%)$ \\
$22(27 \%)$ \\
$19(23 \%)$ \\
$39(47.5 \%)$ \\
$2(2.5 \%)$ \\
$33.6( \pm 5.6)$ \\
$1( \pm 0.08)$ \\
$134( \pm 16)$ \\
$76( \pm 11)$ \\
$9.36(1.3)$ \\
79 \\
$4.5(1.3)$ \\
$2.4(0.9)$ \\
$1(0.2)$ \\
$2.5(1.7)$ \\
$66(81 \%)$ \\
$11(13 \%)$ \\
$5(6 \%)$ \\
$42.2(10.9)$ \\
$47.5(13.1)$ \\
\\
\\
\\
\\
\\
\\
\\
\\
\\
\\
\end{tabular}

\begin{tabular}{|c|c|}
\hline Intervention $(n=83)$ & $P$ value \\
\hline $57.8(9.4)$ & 0.74 \\
\hline $53(64 \%)$ & 0.92 \\
\hline $12.1(6.6)$ & 0.76 \\
\hline $25(30 \%)$ & 0.52 \\
\hline $14(17 \%)$ & 0.71 \\
\hline \multicolumn{2}{|l|}{$32(39 \%)$} \\
\hline $1(1 \%)$ & \\
\hline \multicolumn{2}{|l|}{$36(43 \%)$} \\
\hline $29(35 \%)$ & 0.63 \\
\hline \multicolumn{2}{|l|}{$19(23 \%)$} \\
\hline \multicolumn{2}{|l|}{$42(41 \%)$} \\
\hline \multicolumn{2}{|l|}{$1(1 \%)$} \\
\hline $33.9( \pm 6.2)$ & 0.58 \\
\hline $1( \pm 0.09)$ & 1.00 \\
\hline $133( \pm 14.6)$ & 0.70 \\
\hline $76( \pm 10)$ & 0.74 \\
\hline $9.25(1.2)$ & 0.74 \\
\hline 77 & 0.74 \\
\hline $4.6(1.1)$ & 0.48 \\
\hline $2.6(1.2)$ & 0.31 \\
\hline $1.1(0.3)$ & 0.23 \\
\hline $2.5(1.9)$ & 0.91 \\
\hline \multicolumn{2}{|l|}{$65(78 \%)$} \\
\hline $10(12 \%)$ & 0.69 \\
\hline \multicolumn{2}{|l|}{$8(10 \%)$} \\
\hline $43.6(10.8)$ & 0.44 \\
\hline $45.9(13.7)$ & 0.45 \\
\hline
\end{tabular}

BMI, body mass index; DBP, diastolic blood pressure; HbA1C, glycated hemoglobin; HDL, high-density lipoprotein; LDL, low-density lipoprotein; SBP, systolic blood pressure; S.D., standard deviation; SF-36 v.1, Short Form 36, version 1. 
Table 2 Medication, complications and place of control at baseline.

\begin{tabular}{|c|c|c|c|}
\hline & Control $(n=82)$ & Intervention $(n=83)$ & $P$-value \\
\hline \multicolumn{4}{|l|}{ Medication } \\
\hline Metformin & $61(74 \%)$ & $63(76 \%)$ & 0.96 \\
\hline Sulfonyl urea & $12(15 \%)$ & $12(15 \%)$ & 0.97 \\
\hline GLP-1 analog & $7932(39 \%)$ & $30(36 \%)$ & 0.70 \\
\hline SGLT2 inhibitor & 0 & $1(1.2 \%)$ & 1.00 \\
\hline DPP4-inhibitor & $9(11 \%)$ & $5(6 \%)$ & 0.39 \\
\hline Insulin & $60(73 \%)$ & $57(69 \%)$ & 0.64 \\
\hline $\begin{array}{l}\text { Insulin dose- } \\
\text { mean }\end{array}$ & 74 IE & 73 IE & 0.91 \\
\hline (S.D.; range) & $(48 ; 20-240)$ & $(50 ; 14-240)$ & \\
\hline ACE-I/ARB & $54(66 \%)$ & $48(59 \%)$ & 0.30 \\
\hline \multicolumn{4}{|l|}{ Complications } \\
\hline Microvascular & $64(78 \%)$ & $62(75 \%)$ & 0.61 \\
\hline Macrovascular & $31(38 \%)$ & $17(23 \%)$ & 0.055 \\
\hline None & $15(18 \%)$ & $19(23 \%)$ & 0.47 \\
\hline $\begin{array}{l}\text { Diabetes control } \\
\text { General practice }\end{array}$ & $10(12 \%)$ & $6(7 \%)$ & 0.66 \\
\hline Hospital & $72(88 \%)$ & $77(93 \%)$ & \\
\hline
\end{tabular}

ACE-I, angiotensin-converting factor 1 inhibitor, ARB, angiotensin receptor blocker; DPP4-inhibitor, di-peptidyl-peptidase4-inhibitor; GLP-1, glucagon-like-peptide 1; SGLT2, sodium glucose-co-transporter 2.

the charts of 1151 subjects and randomized 166 of which one was excluded due to screen failure. Baseline data are described in Tables 1 and 2. The mean age of all participants was 58 years (range 30-74), 64\% were males, $28 \%$ had a non-Western background and $23 \%$ were unemployed. The mean HbA1c was 9.3\% (s.D. 1.3) and mean diabetes duration was 12.3 years (s.D. 6.9 , range $1-35)$. The majority (>90\%) attended an outpatient clinic. The remaining participants were in the care of their GP. There were no significant differences at baseline between the control and the telemedicine group (Tables 1 and 2). Participants who discontinued within eight months (prior to completion of the intervention period) were defined as drop outs. Fourteen subjects from the intervention group (17\%) and five from the control group (6\%) dropped out of the study within this period. Seven additional subjects were lost to follow-up (six among controls and one from the intervention group). Overall, $16 \%$ dropped out or were lost to follow-up. This is comparable to other studies and is below the estimated rate of $20 \%$.

Table 3 Mixed-models analysis.

\begin{tabular}{|c|c|c|c|c|}
\hline Type 3 tests of fixed effects & Num DF & Den DF & F Value & Pr $>$ F \\
\hline Inter & 1 & 140 & 9.60 & 0.0023 \\
\hline HbA1c0_cov & 1 & 140 & 88.54 & $<0.0001$ \\
\hline time & 2 & 141 & 0.06 & 0.9405 \\
\hline Age & 1 & 141 & 4.26 & 0.0409 \\
\hline Complications & 3 & 137 & 5.01 & 0.0025 \\
\hline
\end{tabular}

Table 4 Comparison of mean HbA1c difference.

\begin{tabular}{|c|c|c|}
\hline & Estimate & Standard error \\
\hline \multicolumn{3}{|l|}{ Effect } \\
\hline Intervention & -0.4807 & 0.1551 \\
\hline Control & 0 & \\
\hline Baseline $\mathrm{HbA} 1 \mathrm{c}$ & 0.5879 & 0.06248 \\
\hline \multicolumn{3}{|l|}{ Time } \\
\hline 4 months & 0.0258 & 0.07541 \\
\hline 8 months & 0 & \\
\hline Follow-up & 0.0037 & 0.1017 \\
\hline Age & -0.0183 & 0.008879 \\
\hline \multicolumn{3}{|l|}{ Complications } \\
\hline None & -0.6914 & 0.2023 \\
\hline Macrovascular & -0.5642 & 0.5398 \\
\hline Microvascular & 0 & \\
\hline Micro- and macrovascular & -0.4875 & 0.1865 \\
\hline
\end{tabular}

\section{HbA1c}

In the intervention group, $\mathrm{HbA1c}$ was significantly reduced after eight-month intervention $(0.69 \% ; P<0.000001)$ compared to that in control $(0.18 \% ; P=0.22)$. These results were found using a paired $t$-test. Delta values between the two groups were $0.5 \%(P=0.023)$.

In two-way ANOVA, significant effects of intervention $(P=0.003)$, baseline HbA1c $(P<0.01)$ and the interaction between intervention and baseline HbA1c (0.0007) were found. There was no significance of adjusting for age, complications, diabetes duration, sex, education, employment or ethnicity. With mixed-models analysis,

Table 5 Baseline characteristics of drop out vs completers.

\begin{tabular}{|c|c|c|c|}
\hline & $\begin{array}{l}\text { Completed } 32 \text { weeks } \\
\qquad(n=146)\end{array}$ & $\begin{array}{c}\text { Dropout } \\
(n=19)\end{array}$ & $\begin{array}{c}P \\
\text { value }\end{array}$ \\
\hline Age & 58.5 & 54.5 & 0.11 \\
\hline HbA1c & 9.2 & 10 & 0.028 \\
\hline Diabetes duration & 12.5 & 11 & 0.42 \\
\hline BMI & 33.4 & 36.4 & 0.12 \\
\hline $\begin{array}{l}\text { Systolic blood } \\
\text { pressure }\end{array}$ & 133 & 137 & 0.35 \\
\hline $\begin{array}{l}\text { Diastolic blood } \\
\text { pressure }\end{array}$ & 75 & 82 & 0.0506 \\
\hline Hemoglobin & 8.9 & 8.9 & 0.83 \\
\hline Creatinine & 81 & 65 & 0.002 \\
\hline \multicolumn{4}{|l|}{ Education } \\
\hline Higher education & $29(20 \%)$ & $0(0 \%)$ & \\
\hline Skilled & $61(42 \%)$ & $7(37 \%)$ & 0.025 \\
\hline Student & $2(1 \%)$ & $1(5 \%)$ & \\
\hline Unskilled & $54(37 \%)$ & $11(58 \%)$ & \\
\hline \multicolumn{4}{|l|}{ Employment } \\
\hline Employed & $44(30 \%)$ & $7(37 \%)$ & 0.063 \\
\hline Retired & $69(47 \%)$ & $4(21 \%)$ & \\
\hline Student & $2(1 \%)$ & $1(5 \%)$ & \\
\hline Unemployed & $31(22 \%)$ & $7(37 \%)$ & \\
\hline \multicolumn{4}{|c|}{ Short form-36 questionnaire } \\
\hline Physical health & 42.8 & 43.8 & 0.67 \\
\hline Mental health & 47.3 & 42.2 & 0.16 \\
\hline
\end{tabular}


a significant effect of baseline HbA1c $(P<0.0001)$, intervention $(P=0.0023)$, complications $(P=0.0025)$ and age $(P=0.041)$ was found (Tables 3 and 4$)$. High baseline values of HbA1c were associated with greater reductions in HbA1c. Participants with no diabetes complications had the highest reductions in HbA1c followed by participants with macrovascular complications and participants with both microvascular and macrovascular complications. Participants with only microvascular complications had the lowest reductions in HbA1c. Finally, older participants had greater reductions in $\mathrm{HbA1c}$ than younger participants.

No incidents of hypoglycemia were reported. However, due to macrovascular complications, some patients were advised to discuss medication with their doctor due to low HbA1c measured 4 months after randomization. Among patients with macrovascular complications, the mean HbA1c after 4 months was 7.9\% in the intervention group (vs $8.9 \%$ in the control group). The lowest HbA1c was $6.5 \%$ in both intervention and control groups.

In intention-to-treat (ITT) analysis, missing values were imputated using the last measured value. This resulted in a smaller $(0.56 \%)$, but still significant $(P=0.0002)$ reduction in $\mathrm{HbA1c}$ in the telemedicine group. However, the difference between the reduction in HbA1c in the two groups was no longer significant (delta value: $0.39 \%, P=0.055)$.

At six-month follow-up the HbA1c had increased in the intervention group but declined in the control group (+0.18 vs -0.12 ) leaving no difference between the two groups.

\section{Other outcomes}

There were no changes in blood pressure, BMI, waist/hip ratio, lipids, creatinine or fasting glucose in relation to the study. Any significant changes in mental or physical health scores in SF-36 were not encountered. There was no difference in the amount of diabetes controls between the two groups. Diabetes-related admissions and emergency visits were few and only found in the control group. This was not statistically significant. Mean insulin dose was increased in both groups, but there was no difference between the two groups. Subjects who dropped out during the intervention period were characterized by having a higher HbA1c at baseline (10 vs 9.2\%, $P=0.028$ ) and a lower degree of education than subjects who completed the eight-month interventional period $(P=0.039)$ (Table 5). No participants with a higher education dropped out of the study. Creatinine was significantly higher in the completing group $(P=0.002)$. There was a trend toward a lower diastolic blood pressure within the completers $(P=0.051)$. There were no significant differences in age, diabetes duration, sex, ethnicity, SF-36 score or other baseline tests. The withdrawals did not result in any imbalance between the control and telemedicine group.

\section{Discussion and conclusion}

In this randomized study of 165 patients with poorly regulated $\mathrm{T} 2 \mathrm{D}$, we found that video consultations preceded by uploads of relevant measurement as add-on to clinic-based care led to a significant reduction in HbA1c compared to clinic-based care alone. We cannot conclude whether it is the intervention per se or the effect of immediate response to increased measurements, improved compliance and personal contact that lead to the improvement. However, telemedicine is used as a tool for achieving this. The same effect might have been achieved by physical consultations. However, this would have demanded significantly more resources. In the intervention group, HbA1c decreased by $0.69 \%$ compared with baseline levels, whereas the reduction in the control group was $0.18 \%$. However, it was not possible to sustain the improvements in glycemic control at follow-up, six months after intervention. Thus, the effect only lasted when intervention was ongoing. The results are interesting because they show that patients who do not respond to standard efforts can be reached by alternative approaches such as video consultations preceded by uploading of measurements. However, they also suggest that continuing attention is necessary.

The intervention did not have any impact on blood pressure or lipid levels. Even so, the mean baseline values were satisfactory, and hence, no major improvements were expected. We could not detect any differences in BMI, waist/hip ratio, hospital admissions or quality of life assessed by SF-36.

Subjects withdrawing from the study had a higher HbA1c at baseline and a lower degree of education, confirming the social imbalance and underlining the inequality of the health care system. This could imply the need of other kinds of follow-up or additional support for the most vulnerable patients. Limitations of our study include selection bias. Though we presume the effect is mainly due to improved compliance and adherence to treatment. However, we cannot document medical procurement. It could be argued that only the most motivated patients enter a study and that the 
results are consequently not applicable to all patients. However, many of our participants were unemployed, had no education or belonged to ethnic minorities. In other words, many belonged to social groups normally difficult to reach. Blinding of outcome assessors for randomization status would have been preferable and would have enhanced the study's validity. The study was cross-sectorial using health care center nurses in the video consultations. These nurses did not have the authorization to prescribe medicine. Hence, medication management was not a possibility.

\section{Strengths and weaknesses of the study}

Regulating medication - especially insulin - during the video consultations would probably have resulted in a more pronounced effect. However, the study was designed to enhance empowerment-based education by use of telemedicine. Furthermore, the increased number of contacts with a health care professional in itself could improve the glycemic regulation. However, one of the advantages of telehealth is the possibility of increasing the number of consultations without forcing the patients to go to a health care clinic. Our main interest was to reach patients who did not respond to standard regimens. We found a significant reduction in $\mathrm{HbA1c}$, and in contrast to most other studies, our study examined intervention as add-on to clinic-based care and was not performed in a medically underserved area. Thus, our focus was on reaching patients who could not be reached despite offers of high quality of care.

A systematic review including RCTs where HbA1c was the primary outcome compared intervention to usual care and found a positive effect of the different teleinterventions in general (25). However, the authors conclude the need of individualized and holistic intervention and of approaches focusing on promoting self-care and proactive collaborations between health care professionals and patients to manage diabetes care. Our study was a collaboration between the hospital and the municipality. The nurses were trained using empowerment-based conversations, and although conversations were based upon an interview guide, the conversation was individualized according to the needs of the patients. Given a more extended collaboration between the nurses and the specialist, even better results may have been achieved. Most studies compared intervention to clinic-based care $(21,25,26,27,28,29$,
30, 31, 32, 33, 34). Among these, the Whole System Demonstrator (WSD) found a positive effect on HbA1c, but no effect on quality of life (30). An Italian multicentre study found a significant reduction of HbA1c and an improvement in Qol but in line with our study, they found no effect on lipids, blood pressure and weight (35). A Norwegian study tested a mobile phone app as add-on to usual care, finding no difference in HbA1c between the groups after one year (27). In contrast to other studies, intervention consisted only of telephone consultations, and there were no visual contacts.

The longest reported telemedicine study is the IDEATel. Here, significant reductions in $\mathrm{HbA1c}$, blood pressure and LDL cholesterol were seen in years 1,4 and $5(21,36)$. However, this study was done in a medically underserved area, and intervention was used as a replacement for a low level of standard care. In contrast, our participants were not medically underserved, but patients who had not responded to standard treatment. Clinic-based care in Denmark offers all patients with type 2 diabetes a diabetes management program - doctors' visit every 3-6 months and perform annual screening for complications. Moreover, annual nurse visits for all patients followed in a diabetes outpatient clinic (90\% of the participants). Thus, we focused on a population that was offered a high level of standard care including a diabetes management program, but who due to life circumstances could not benefit from it.

Social inequality in the health care system is well known, and vulnerable patients can be difficult to reach. Many of our patients could be characterized as being vulnerable due to comorbidity, lack of employment (23\%) and education (39\% overall). All factors associated with higher mortality and a higher risk of developing complications $(3,6)$. In the current study, we examined the effect of education, ethnicity and employment finding these factors did not influence baseline values or the study outcome. Thus, vulnerability may not solely be due to SES but life circumstances per se. In the IDEATel, Hispanics had the highest baseline A1C levels and showed the greatest improvement in the intervention (17). We also found greater reductions among patients with high baseline levels of HbA1c but no differences between ethnical groups.

In the United States, Hispanics often represent a lower social group. Thus, the difference between ethnic groups found in the IDEATel study may mainly be due to social groups rather than ethnicity. Most of our participants were socially deprived. This could explain why we did not see the same differences in ethnic groups. 
We found $16 \%$ of the participants dropped out of the study. Sanders and coworkers examined barriers for telehealth doing semi-structured interviews of 22 people who declined to participate in or dropped out of the Whole Systems Demonstrator (WSD). They found that concerns of need for special skills, fear of threats to identity and expectations and experiences of disruption to services were associated with declining participation or dropping out of the study (37). We have not done systematic interviews with the non-completers, but we found that participants dropping out had higher HbA1c levels and a lower degree of education than those completing. Moreover, no participants with high education dropped out. Thus, although we reached a vulnerable population, it seems we were still not able to reach the most vulnerable patients. This stresses the importance of differentiating treatment as telehealth intervention might not be appropriate for some of the less-educated patients. Furthermore, it stresses the problem of social imbalance. In our study, tablets and glucose meters were handed out to participants in the intervention group. Today, many currently used glucose meters can upload data online. Moreover, the use of smart phones has increased significantly enabling the communication via Skype or similar programs. Thereby, even without the customized software used in this study, it would be possible to reproduce the intervention. However, when implementing in practice, it would be preferable with a number of designated loaner tablets for patients thereby overcoming the economic difficulties and disparities.

\section{Conclusion}

Taken together, our study gives evidence that telemedicine as a tool for ensuring regular measurements and feedback can lead to clinically significant improvements in glycemic control among patients who have not responded to standard regimens. However, continuing effort and attention are essential as the effect does not persist when intervention ends. Moreover, the low adherence in the subgroup with very poor glycemic regulation and low education indicates that our setup could be too difficult or that other circumstances in life outweigh the concern for diabetes implying the need for specially tailored solutions for some patients. Future studies should focus on differentiation substantiating how to meet these patients' needs as alternative approaches have yet to be encountered. Moreover, further studies are essential for addressing the issue of non-compliance and nonattendance.
Declaration of interest

The authors declare that there is no conflict of interest that could be perceived as prejudicing the impartiality of this study.

\section{Funding}

The study was supported by the Capital Region of Denmark and the City of Copenhagen. Furthermore, the study was supported by and by a grant from 'Smedemester Niels Hansens og hustru Frederikkes' Fund.

\section{Author contribution statement}

All authors participated in the design of the study and provided input into the main ideas of this paper. C R Hansen enrolled participants, performed the statistical analyses and drafted the manuscript. All authors read, commented and approved the final version of the manuscript.

\section{Acknowledgements}

The authors thank all participants for their engagement, patience and participation in the study. Furthermore, they thank Merete Halkjær, Jens Egsgaard and Pernille Faurschou, Health and Care Administration, City of Copenhagen, Dorte Høst, Department of Integrated Care, Bispebjerg Hospital, Henriette Schnedler, Department of Endocrinology, Bispebjerg Hospital, Christina Hornbøll Jørgensen, Anne Kulmann Jensen, Lisbeth Jul Olsen and Anne Kristine Hvas van der Zaag, Healthcare Center Noerrebro, City of Copenhagen. Finally, they thank Bayer Healthcare for support of glucose meters and test strips.

\section{References}

1 IDF Diabetes Atlas 2014, 2015.

2 Connolly V, Unwin N, Sherriff P, Bilous R \& Kelly W. Diabetes prevalence and socioeconomic status: a population based study showing increased prevalence of type 2 diabetes mellitus in deprived areas. Journal of Epidemiology and Community Health 200054 173-177. (doi:10.1136/jech.54.3.173)

3 Espelt A, Borrell C, Roskam AJ, Rodríguez-Sanz M, Stirbu I, mauBueno A, Regidor E, Bopp M, Martikainen P, Leinsalu M et al. Socioeconomic inequalities in diabetes mellitus across Europe at the beginning of the 21st century. Diabetologia 200851 1971-1979.

4 Gnavi R, Karaghiosoff L, Costa G, Merletti F \& Bruno G. Socioeconomic differences in the prevalence of diabetes in Italy: the population-based Turin study. Nutrition, Metabolism and Cardiovascular Diseases 200818 678-682. (doi:10.1016/j.numecd.2007.11.004)

5 Sacerdote C, Ricceri F, Rolandsson O, Baldi I, Chirlaque MD, Feskens E, Bendinelli B, Ardanaz E, Arriola L, Balkau B et al. Lower educational level is a predictor of incident type 2 diabetes in European countries: the EPIC-InterAct study. International Journal of Epidemiology 201241 1162-1173. (doi:10.1093/ije/dys091)

6 Grintsova O, Maier W \& Mielck A. Inequalities in health care among patients with type 2 diabetes by individual socio-economic status (SES) and regional deprivation: a systematic literature review. International Journal for Equity in Health 20141343. (doi:10.1186/1475-9276-13-43)

7 Jenum AK, Diep LM, Holmboe-Ottesen G, Holme IM, Kumar BN \& Birkeland KI. Diabetes susceptibility in ethnic minority groups from Turkey, Vietnam, Sri Lanka and Pakistan compared with Norwegians the association with adiposity is strongest for ethnic minority women. BMC Public Health 201212 150. (doi:10.1186/1471-2458-12-150)

8 Shai I, Jiang R, Manson JE, Stampfer MJ, Willett WC, Colditz GA \& $\mathrm{Hu}$ FB. Ethnicity, obesity, and risk of type 2 diabetes in women: a 20-year follow-up study. Diabetes Care 200629 1585-1590. (doi:10.2337/dc06-0057) 
9 Chatterji P, Joo H \& Lahiri K. Racial/ethnic- and education-related disparities in the control of risk factors for cardiovascular disease among individuals with diabetes. Diabetes Care 201235 305-312. (doi:10.2337/dc11-1405)

10 Dinesen C, Nielsen SS, Mortensen LH \& Krasnik A. Inequality in selfrated health among immigrants, their descendants and ethnic Danes: examining the role of socioeconomic position. International Journal of Public Health 201156 503-514. (doi:10.1007/s00038-011-0264-6)

11 Nielsen SS \& Krasnik A. Poorer self-perceived health among migrants and ethnic minorities versus the majority population in Europe: a systematic review. International Journal of Public Health $2010 \mathbf{5 5}$ 357-371. (doi:10.1007/s00038-010-0145-4)

12 Philis-Tsimikas A, Fortmann A, Lleva-Ocana L, Walker C \& Gallo LC. Peer-led diabetes education programs in high-risk Mexican Americans improve glycemic control compared with standard approaches: a Project Dulce promotora randomized trial. Diabetes Care 201134 1926-1931. (doi:10.2337/dc10-2081)

13 Philis-Tsimikas A, Walker C, Rivard L, Talavera G, Reimann JO, Salmon M \& Araujo R. Improvement in diabetes care of underinsured patients enrolled in project dulce: a community-based, culturally appropriate, nurse case management and peer education diabetes care model. Diabetes Care 200427 110-115. (doi:10.2337/ diacare.27.1.110)

14 Walker EA, Shmukler C, Ullman R, Blanco E, Scollan-Koliopoulus M \& Cohen HW. Results of a successful telephonic intervention to improve diabetes control in u rban adults: a randomized trial. Diabetes Care $2011342-7$.

15 American Telemedicine Association, 2012. http://www. americantelemed.org/about/telehealth-faqs-

16 Shea S, Kothari D, Teresi JA, Kong J, Eimicke JP, Lantigua RA, Palmas W $\&$ Weinstock RS. Social impact analysis of the effects of a telemedicine intervention to improve diabetes outcomes in an ethnically diverse, medically underserved population: findings from the IDEATel Study. American Journal of Public Health 2013103 1888-1894.

17 Weinstock RS, Teresi JA, Goland R, Izquierdo R, Palmas W, Eimicke JP, Ebner S \& Shea S. Glycemic control and health disparities in older ethnically diverse underserved adults with diabetes: five-year results from the Informatics for Diabetes Education and Telemedicine (IDEATel) study. Diabetes Care 201134 274-279.

18 Fatehi F, Gray LC \& Russell AW. Telemedicine for clinical management of diabetes - a process analysis of video consultations. Journal of Telemedicine and Telecare 201319 379-382.

19 Izquierdo RE, Knudson PE, Meyer S, Kearns J, Ploutz-Snyder R \& Weinstock RS. A comparison of diabetes education administered through telemedicine versus in person. Diabetes Care 200326 1002-1007. (doi:10.2337/diacare.26.4.1002)

20 Jia H, Chuang HC, Wu SS, Wang X \& Chumbler NR. Long-term effect of home telehealth services on preventable hospitalization use. Journal of Rehabilitation Research and Development 200446 557-566. (doi:10.1682/JRRD.2008.09.0133)

21 Shea S, Weinstock RS, Starren J, Teresi J, Palmas W, Field L, Morin P, Goland R, Izquierdo RE, Wolff LT et al. A randomized trial comparing telemedicine case management with usual care in older, ethnically diverse, medically underserved patients with diabetes mellitus. Journal of the American Medical Informatics Association 200613 40-51.

22 Snorgaard O. NBV: Behandling og kontrol af type 2 diabetes, 2014. http://www.endocrinology.dk/PDF/ FarmakologiskbehandlingDM2rev2014.pdf.

23 Hansen CR, Perrild H, Koefoed BG, Faurschou P, Host D \& Zander M. Effects of telemedicine in the treatment of patients with type 2 diabetes - a study protocol. Danish Medical Journal 201360 A4743.
24 Gerstein HC, Miller ME, Genuth S, Ismail-Beigi F, Buse JB, Goff DC Jr, Probstfield JL, Cushman WC, Ginsberg HN, Bigger JT et al. Long-term effects of intensive glucose lowering on cardiovascular outcomes. New England Journal of Medicine 2011364 818-828.

25 Verhoeven F, Tanja-Dijkstra K, Nijland N, Eysenbach G \& van GemertPijnen L. Asynchronous and synchronous teleconsultation for diabetes care: a systematic literature review. Journal of Diabetes Science and Technology 20104 666-684.

26 Anderson DR, Christison-Lagay J, Villagra V, Liu H \& Dziura J. Managing the space between visits: a randomized trial of disease management for diabetes in a community health center. Journal of General Internal Medicine 201025 1116-1122. (doi:10.1007/s11606010-1419-5)

27 Holmen H, Torbjornsen A, Wahl AK, Jenum AK, Smastuen MC, Arsand E \& Ribu L. A mobile health intervention for self-management and lifestyle change for persons with type 2 diabetes, part 2: one-year results from the norwegian randomized controlled trial RENEWING HEALTH. JMIR mHealth and uHealth 20142 e57. (doi:10.2196/mhealth.3882)

28 Pressman AR, Kinoshita L, Kirk S, Barbosa GM, Chou C \& Minkoff J. A novel telemonitoring device for improving diabetes control: protocol and results from a randomized clinical trial. Telemedicine and E-Health 201420 109-114

29 Rasmussen OW, Lauszus FF \& Loekke M. Telemedicine compared with standard care in type 2 diabetes mellitus: a randomized trial in an outpatient clinic. Journal of Telemedicine and Telecare 201622 363-368. (doi:10.1177/1357633x15608984)

30 Steventon A, Bardsley M, Billings J, Dixon J, Doll H, Hirani S, Cartwright M, Rixon L, Knapp M, Henderson C et al. Effect of telehealth on use of secondary care and mortality: findings from the Whole System Demonstrator cluster randomised trial. BMJ 2012344 e3874. (doi:10.1136/bmj.e3874)

31 Wakefield BJ, Koopman RJ, Keplinger LE, Bomar M, Bernt B, Johanning JL, Kruse RL, Davis JW, Wakefield DS \& Mehr DR. Effect of home telemonitoring on glycemic and blood pressure control in primary care clinic patients with diabetes. Telemedicine and E-Health 201420 199-205.

32 Whitlock WL, Brown A, Moore K, Pavliscsak H, Dingbaum A, Lacefield D, Buker K \& Xenakis S. Telemedicine improved diabetic management. Military Medicine 2000165 579-584.

33 Zhai YK, Zhu WJ, Cai YL, Sun DX \& Zhao J. Clinical- and costeffectiveness of telemedicine in type 2 diabetes mellitus: a systematic review and meta-analysis. Medicine 201493 e312.

34 Zhou P, Xu L, Liu X, Huang J, Xu W \& Chen W. Web-based telemedicine for management of type 2 diabetes through glucose uploads: a randomized controlled trial. International Journal of Clinical and Experimental Pathology 20147 8848-8854.

35 Nicolucci A, Cercone S, Chiriatti A, Muscas F \& Gensini G. A randomized trial on home telemonitoring for the management of metabolic and cardiovascular risk in patients with type 2 diabetes. Diabetes Technology and Therapeutics 201517 563-570. (doi:10.1089/dia.2014.0355)

36 Shea S, Weinstock RS, Teresi JA, Palmas W, Starren J, Cimino JJ, Lai AM, Field L, Morin PC, Goland R et al. A randomized trial comparing telemedicine case management with usual care in older, ethnically diverse, medically underserved patients with diabetes mellitus: 5 year results of the IDEATel study. Journal of the American Medical Informatics Association 200916 446-456. (doi:10.1197/jamia.M3157)

37 Sanders C, Rogers A, Bowen R, Bower P, Hirani S, Cartwright M, Fitzpatrick R, Knapp M, Barlow J, Hendy J et al. Exploring barriers to participation and adoption of telehealth and telecare within the Whole System Demonstrator trial: a qualitative study. BMC Health Services Research 201212 220. (doi:10.1186/1472-6963-12-220)
Received 28 September 2016

Revised version received 15 March 2017

Accepted 20 March 2017 